S1.6

\section{ESPERIENZE NELLA GESTIONE DI EPIDEMIE NOSOCOMIALI: IRCCS SAN MATTEO DI PAVIA}

\section{Marone P., Carretto E.}

Laboratorio di Batteriologia, Laboratori Sperimentali di

Ricerca, Area Infettivologica, IRCCS Policlinico

"San Matteo", Pavia

Gli stafilococchi aurei resistenti alla meticillina (SAMR) sono fra i patogeni più frequentemente isolati nelle Terapie Intensive (TI). Tra il luglio 1998 e il giugno 1999 è stato messo in atto un protocollo di sorveglianza sulle colonizzazioni/infezioni da SAMR presso una delle 3 TI dell'IRCCS Policlinico "San Matteo" di Pavia. Sono stati arruolati nello studio 292 pazienti, 87 dei quali sono risultati colonizzati (29.8\%). In 40/87 (13.7\%) la colonizzazione era già presente all'ingresso, mentre in 47/87 (18.6\%) è avvenuta in TI. Nell'ambito dei pazienti colonizzati, 26/87 (29.8\%) hanno sviluppato un'infezione da SAMR. Analisi molecolari (ribotipizzazione con sistema automatico) hanno consentito di stimare dodici differenti cluster di microrganismi, il più numeroso dei quali risultava essere composto da un elevato numero di isolati (146).

Nel periodo compreso fra l'1 gennaio 1997 e il 31 dicembre 2001 è stato condotto uno studio di sorveglianza relativamente agli enterobatteri produttori di beta-lattamasi a spettro allargato (ESBL+) isolati da una TI del nostro ospedale. P. mirabilis è risultato il microrganismo che più frequentemente ha mostrato il fenotipo ESBL+ (58\%). In 312 casi l'isolamento dell'enterobattere è stato considerato come causa di infezione; nell'ambito dei diversi episodi infettivi, i ceppi $\mathrm{ESBL}+$ sono risultati essere causa di infezioni delle vie urinarie nel $44.9 \%$ dei casi, di sepsi nel $30 \%$, di polmoniti nel $25.8 \%$, di infezioni di ferita nel $20.4 \%$. La mortalità complessiva nelle infezioni sostenute da ceppi ESBL+ è risultata essere pari all' $1 \%$, contro il $10.6 \%$ dei ceppi non produttori. Nel periodo considerato, l'incidenza dei ceppi ESBL+ è andata progressivamente riducendosi, passando dal 38.9\% del 1997 al $10.9 \%$ del 2001. Questo risultato è stato ottenuto grazie alle misure messe in atto nell'U.O. (precauzioni di barriera, variazioni nella terapia antibiotica empirica ecc.).

Dal giugno 1998, a seguito del primo caso di sepsi da enterococchi resistenti alla vancomicina (VRE) presso il nostro Ospedale, è in atto un protocollo di sorveglianza attiva delle infezioni/colonizzazioni da VRE. Le U.O. coinvolte sono state sinora le 3 TI e l'Oncoematologia Pediatrica, anche se isolamenti sporadici di questo microrganismo sono stati osservati anche in altri Reparti. Un outbreak di colonizzazioni si è avuto presso una delle TI: a partenza da un caso indice, sei pazienti degli 8 ricoverati sono risultati colonizzati da VRE. Da allora e nei 16 mesi successivi sono stati analizzati i tamponi rettali di 509 pazienti. Di essi $13(2.5 \%)$ sono risultati colonizzati all'ingresso in TI, mentre $43(8.7 \%)$ dei restanti 496 pazienti VRE negativi hanno acquisito il microrganismo durante la degenza in TI. Il fattore di rischio più evidente è risultato essere, nella nostra esperienza, la durata della degenza in TI. Due pazienti hanno sviluppato infezione da VRE; l'indice colonizzazioni/infezioni è quindi risultato essere del $3.6 \%$ e la frequenza complessiva delle infezioni da VRE dello 0.4\%. Entrambi i pazienti, trattati con oxazolidinoni, sono guariti. Sui diversi ceppi è stata eseguita ribotipizzazione con sistema automatico, utilizzando differenti enzimi di restrizione, che ha consentito di identificare tre differenti cluster: al più numeroso erano ascrivibili 52 pazienti, mentre altri due, uno dei quali causato da ceppi di $E$. faecalis (mentre tutti gli altri erano E.faecium), sono risultati essere composti da due pazienti. A seguito del manifestarsi dell'epidemia sono state messe in atto procedure di isolamento e training del personale come indicato nelle procedure dei Centers for Disease Control del 1995. L'incidenza degli isolamenti si è progressivamente ridotta nel mesi successivi, con un caratteristico andamento "a dente di sega" e con mesi liberi da isolamenti a partire dal $16^{\circ}$ successivamente all'outbreak iniziale. Peraltro il ripresentarsi di nuovi casi sporadici nel reparto di TI sembrerebbe documentare uno stato di endemia a livello del nostro Ospedale, rilevabile più facilmente nelle aree critiche quali le TI che hanno uno scambio continuo di pazienti con altre unità operative.

\section{S1.7}

\section{ESPERIENZE NELLA GESTIONE DI EPIDEMIE NOSOCOMIALI: POLICLINICO UNIVERSITARIO DI PARMA}

\section{Menozzi M.G., Bertoncini L., Zerbini L., Rossi S., Somenzi P., Chezzi C., Dettori G. \\ Sezione di Microbiologia, Dip. di Patologia e Medicina di Laboratorio-Univ. degli Studi di Parma}

Clostridium difficile enterotossinogenico è responsabile di un ampio spettro di infezioni: una colonizzazione asintomatica, una diarrea autolimitantesi, coliti di varia gravità fino alla colite pseudomembranosa. Rappresenta inoltre la principale causa di diarree nosocomiali, problema di grande rilevanza socio-economica negli ambienti ospedalieri. La popolazione maggiormente suscettibile alla malattia è rappresentata dai 